\title{
Impact of nonabsorbing anthropogenic aerosols on clear-sky atmospheric absorption
}

\author{
Philip Stier, ${ }^{1}$ John H. Seinfeld, ${ }^{2}$ Stefan Kinne, ${ }^{3}$ Johann Feichter, ${ }^{3}$ and Olivier Boucher ${ }^{4}$ \\ Received 1 February 2006; revised 25 May 2006; accepted 14 June 2006; published 19 September 2006.
}

[1] Absorption of solar radiation by atmospheric aerosol has become recognized as important in regional and global climate. Nonabsorbing, hydrophilic aerosols, such as sulfate, potentially affect atmospheric absorption in opposing ways: first, decreasing absorption through aging initially hydrophobic black carbon (BC) to a hydrophilic state, enhancing its removal by wet scavenging, and consequently decreasing $\mathrm{BC}$ lifetime and abundance, and second, increasing absorption through enhancement of the $\mathrm{BC}$ absorption efficiency by internal mixing as well as through increasing the amount of diffuse solar radiation in the atmosphere. On the basis of General Circulation Model studies with an embedded microphysical aerosol module we systematically demonstrate the significance of these mechanisms both on the global and regional scales. In remote transport regions, the first mechanism prevails, reducing atmospheric absorption, whereas in the vicinity of source regions, despite enhanced wet scavenging, absorption is enhanced owing to the prevalence of the second mechanisms. Our findings imply that the sulfur to $\mathrm{BC}$ emission ratio plays a key role in aerosol absorption.

Citation: Stier, P., J. H. Seinfeld, S. Kinne, J. Feichter, and O. Boucher (2006), Impact of nonabsorbing anthropogenic aerosols on clear-sky atmospheric absorption, J. Geophys. Res., 111, D18201, doi:10.1029/2006JD007147.

\section{Introduction}

[2] Atmospheric aerosols play an important role in the global climate system through direct modification of the global radiation budget, by scattering and absorption, as well as indirectly by the modification of cloud properties. Early studies of the direct aerosol effects focused on pure sulfate (SU) aerosol that predominantly scatters solar radiation and therefore leads to cooling [e.g., Langner and Rodhe, 1991; Kiehl and Briegleb, 1993; Feichter et al., 1996]. More recently, the importance of other anthropogenic aerosol components, in particular of carbonaceous aerosols, has received considerable attention [e.g., Cooke et al., 1999; Penner et al., 2001; Chung and Seinfeld, 2005] and their relative importance is projected to increase according to emission scenarios [Nakicenovic, 2000]. Unlike pure sulfate, black carbon (BC), because of its high absorptivity in the visible wavelengths, acts predominantly to warm the atmosphere [Charlson and Pilat, 1969; Penner et al., 2001; Chung and Seinfeld, 2002]. Contrary to scattering aerosols that to a first order only affect the atmospheric heat balance via modification of the surface fluxes, absorbing aerosols in

\footnotetext{
${ }^{1}$ Department of Environmental Science and Engineering, California Institute of Technology, Pasadena, California, USA.

${ }^{2}$ Department of Chemical Engineering and Department of Environmental Science and Engineering, California Institute of Technology, Pasadena, California, USA.

${ }^{3}$ Atmosphere in the Earth System, Max Planck Institute for Meteorology, Hamburg, Germany.

${ }^{4}$ Hadley Centre, Met Office, Exeter, UK.

Copyright 2006 by the American Geophysical Union. 0148-0227/06/2006JD007147\$09.00
}

addition directly heat the atmosphere with potentially strong impacts for regional climate [Menon et al., 2002; Roeckner et al., 2006].

[3] The sign and magnitude of direct aerosol radiative effects depend not only on the spatial distribution of the aerosol and the aerosol size distribution but also on its mixing state. Internal mixing, i.e., chemical components occur mixed in each individual particle, has shown to have the potential to enhance aerosol absorption over external mixing, in which each component occurs in independent particles [Ackerman and Toon, 1981; Chýlek et al., 1995; Jacobson, 2000]. The geometrical arrangement of the aerosol components in a particle influences the mass absorption efficiency, increasing from an internally mixed concentric core-shell alignment to that for the fully internally mixed case. A number of studies have investigated the effect of internal mixing on aerosol radiative properties and effects [Chýlek et al., 1995; Martins et al., 1998a; Jacobson, 2001; Lesins et al., 2002; Myhre et al., 2004; Chung and Seinfeld, 2005; Schnaiter et al., 2005]. In summary, these studies have concluded that the internal mixture of $\mathrm{BC}$ with other aerosol components increases the absorption efficiency, i.e., co-single scattering albedo, of the aerosol population and consequently increases the overall atmospheric absorption.

[4] Internal mixing also affects aerosol microphysical properties. The conversion of initially hydrophobic particles to a hydrophilic state, enhancing the scavenging efficiency and consequently decreasing the aerosol lifetime, is commonly approached in the context of aerosol aging. These aging processes are of particular importance for $\mathrm{BC}$ that is generally assumed to be in a hydrophobic state upon emission from combustion processes [Weingartner et al., 
1997]. A number of studies investigating the effects of aerosol aging have assumed either fixed aging times [Cooke et al., 1999; Koch, 2001] or adapted a mechanistic approach [Riemer et al., 2004; Stier et al., 2006a; Croft et al., 2005; Stier et al., 2006b]. In summary, these studies concluded that the presence of hydrophilic nonabsorbing aerosol species, such as SU, enhances the microphysical aging of $\mathrm{BC}$ to a hydrophilic state. Consequently, the atmospheric lifetime of $\mathrm{BC}$ is reduced, since, associated with being hydrophilic, it is now subject to more efficient wet removal. With more efficient removal, the atmospheric burden of $\mathrm{BC}$ is reduced, along with its associated absorption.

[5] In addition, the presence of scattering aerosols in the atmosphere, such as SU, affects the amount of solar radiation available for absorption by aerosols and gases.

[6] These counteracting mechanisms of nonabsorbing aerosols on atmospheric absorption have not been investigated in a systematic manner. Here we investigate through General Circulation Model studies the global and regional significance of the interaction between anthropogenic sulfate and black carbon on direct aerosol radiative forcing, with a particular focus on atmospheric absorption. As anthropogenic aerosols interact primarily with the shortwave part of the spectrum, longwave radiative effects are neglected.

\section{Model Description}

[7] We perform simulations for the year 2000 with the aerosol-climate model ECHAM5-HAM comprising the ECHAM5 atmospheric general circulation model and the microphysical aerosol module HAM.

\subsection{ECHAM5 General Circulation Model}

[8] ECHAM5 [Roeckner et al., 2003] is the fifth-generation climate model developed at the Max Planck Institute for Meteorology, evolving from the model of the European Centre for Medium-Range Weather Forecasts. ECHAM5 solves prognostic equations for vorticity, divergence, surface pressure and temperature expressed in terms of spherical harmonics with a triangular truncation. Water vapor, cloud liquid water, cloud ice and trace components are transported with a flux form semi-Lagrangian transport scheme [Lin and Rood, 1996] on a Gaussian grid. ECHAM5 contains a microphysical cloud scheme [Lohmann and Roeckner, 1996] with prognostic equations for cloud liquid water and ice. Cloud cover is predicted with a prognostic-statistical scheme solving equations for the distribution moments of total water [Tompkins, 2002]. Convective clouds and convective transport are based on the mass flux scheme of Tiedtke [1989] with modifications by Nordeng [1994]. The solar radiation scheme, updated from Fouquart and Bonnel [1980], has 4 spectral bands, 1 for the visible and ultraviolet, and 3 for the near-infrared. The longwave radiation scheme [Mlawer et al., 1997; Morcrette et al., 1998] has 16 spectral bands. ECHAM5 has the capability to perform nudged simulations, i.e., to relax the prognostic variables toward an atmospheric reference state, such as forecast or reanalysis data from operational weather forecast models. A resolution of horizontally T63 (corresponding to $1.8^{\circ}$ ) with 31 vertical levels is applied for this study.
Table 1. Modal Structure of the Aerosol Module HAM Where SU (Sulfate), BC (Black Carbon), POM (Particulate Organic Matter), SS (Sea Salt), DU (Dust) Denote the Consideration of the Component in the Respective Mode [Stier et al., 2005]

\begin{tabular}{lll}
\hline & \multicolumn{1}{c}{ Hydrophilic } & Hydrophobic \\
\hline Nucleation & SU & \\
Aitken & SU, BC, POM & BC, POM \\
Accumulation & SU, BC, POM, SS, DU & DU \\
Coarse & SU, BC, POM, SS, DU & DU \\
\hline
\end{tabular}

\subsection{Aerosol Module HAM}

[9] The microphysical aerosol module HAM [Stier et al., 2005] incorporates the dominant global aerosol components: sulfate (SU), black carbon (BC), particulate organic matter (POM), sea salt, and mineral dust. The aerosol size distribution and mixing state are represented by the superposition of seven partly internally mixed modes. The composition of each mode is prognostic within the allowed mixing state configuration (Table 1) and determined by the governing microphysical processes of coagulation, condensation, and water uptake [see Vignati et al., 2004; Stier et al., 2005]. BC is emitted into the internally mixed hydrophobic BC/POM Aitken mode and transferred by microphysical aging, i.e., the coagulation with hydrophilic modes and the condensation of sulfuric acid, to internally mixed hydrophilic Aitken, accumulation, and coarse modes. Once hydrophobic particles are coated with a monolayer of sulfate or coagulate with hydrophilic particles, they are transferred to the hydrophilic mode of the same size class or the larger size class for the case of coagulation of unequally sized particles. In the standard version of HAM, effective refractive indices for the internally mixed modes are calculated on the basis of volume averaging of the refractive indices of all components, including aerosol water [Stier et al., 2005]. Refractive indices for sulfate $\left(1.43-10^{-8} \mathrm{i}\right.$ at $\left.550 \mathrm{~nm}\right)$ and black carbon $(1.75-0.44 \mathrm{i}$ at $550 \mathrm{~nm}$ ) are based on Hess et al. [1998]. As the volume averaging approach has the tendency to overestimate the imaginary part of the effective refractive index for the case of strongly absorbing inclusions, such as BC, in a less absorbing host medium, such as sulfate-water mixtures, additional sensitivity simulations employing the Bruggeman [1935] mixing rule for effective refractive indices (see Appendix A) are performed. Aerosol radiative properties for each mode are calculated interactively from the mode size distribution and effective refractive index and passed to the ECHAM5 radiation scheme. For wet deposition, differentiation between the hydrophobic and hydrophilic modes is represented in the form of mode-specific semiempirical scavenging parameters, with reduced scavenging ratios for the hydrophobic modes [see Stier et al., 2005, Table 3]. The assumption of a residual scavenging efficiency of the hydrophobic modes accounts for unresolvable subgrid-scale aging processes [Jacobson and Seinfeld, 2004] and for the neglect of chemical aging processes [Zuberi et al., 2005]. Additional sensitivity studies are performed with the assumption of identical scavenging efficiency for the hydrophobic modes as for the hydrophilic modes of the same size class, and with the assumption of zero scavenging efficiency of the hydrophobic modes. These two assumptions can be 
a)

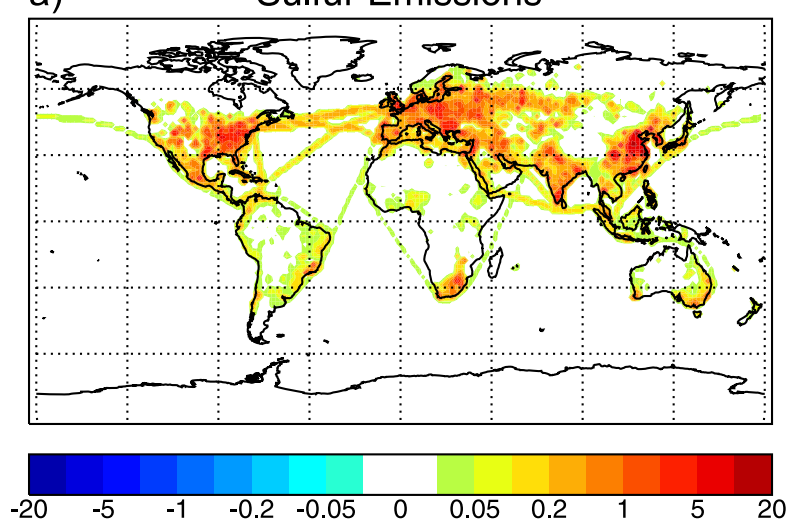

b) Sulfur Emissions

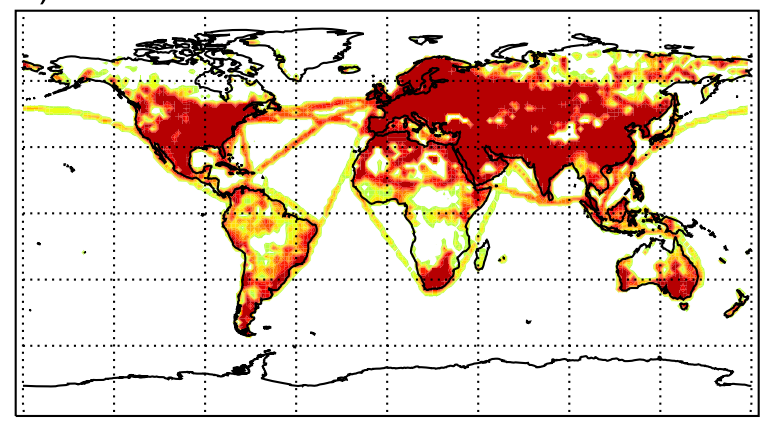

$-100 \%-80 \%-60 \% \quad-40 \% \quad-20 \% \quad 0 \% \quad 20 \% \quad 40 \% \quad 60 \% \quad 80 \% \quad 100 \%$

Figure 1. Annual (a) total anthropogenic sulfur emissions $\left(\mathrm{g} \mathrm{m}^{-2} \mathrm{yr}^{-1}\right)$ and (b) percent contribution of anthropogenic to total sulfur year 2000 emissions (\%), i.e., difference between year 2000 case with all emissions and an identical case without $\mathrm{SO}_{2}$ emissions from fossil fuel use, industry, and biofuels.

seen as the respective upper and lower bounds for the influence of nonabsorbing hydrophilic anthropogenic aerosols on hydrophobic aerosols.

\subsection{Simulation Setup}

[10] The effects of anthropogenic sulfate are isolated as the difference between a year 2000 simulation with the complete year 2000 emissions and an identical simulation without $\mathrm{SO}_{2}$ emissions from fossil fuel use, industry, and biofuels, which comprise $54.2 \mathrm{Tg}(\mathrm{S})$ out of total emissions of $94.6 \mathrm{Tg}(\mathrm{S})$. The reference simulation with the complete year 2000 emissions is identical to the comprehensively evaluated simulation by Stier et al. [2005]. Emissions of mineral dust, sea salt, and marine dimethylsulfide are calculated interactively; all other emissions are based on the year 2000 AeroCom emission inventory [Dentener et al., 2006] (available at ftp://ftp.ei.jrc.it/pub/Aerocom/). The large-scale meteorology in all simulations is constrained by the nudging technique to year 2000 ECMWF ERA-40 reanalysis data [Simmons and Gibson, 2000]. Thus the aerosol radiative effects on the meteorology are small and confined to local scales. Radiative forcing is calculated as the difference in the respective net radiative fluxes from two nudged simulations in only one of which the respective perturbation is included [see Stier, 2005].

\section{Results}

\subsection{Reference Scenario}

[11] The addition of anthropogenic sulfur, which dominates total sulfur emissions in the major source regions (Figures 1a and 1b), to all other emissions increases the annual mean column burden of SU in the anthropogenic source and export regions of the Northern Hemisphere shown in Figures 2a and 2b. However, as is evident in Figure $2 \mathrm{c}$, the presence of anthropogenic sulfur emissions also leads to a distinct reduction of the $\mathrm{BC}$ burden in the source and export regions from that in the absence of anthropogenic sulfur emissions. Relative changes with respect to the year 2000 case with all emissions (Figure 2d) show that the year $2000 \mathrm{BC}$ burden is reduced by about $10 \%$ to $20 \%$ in the source and by about $50 \%$ to $100 \%$ in the remote transport regions North Pacific, North Atlantic, and North Polar region. This reduction results from the intensification of microphysical aging of initially emitted hydrophobic BC owing to both the condensation of sulfuric acid and coagulation with hydrophilic SU containing aerosols. By including anthropogenic sulfur emissions, the global annual mean microphysical aging time of $\mathrm{BC}$, defined as the ratio of the mass burden of the hydrophobic fraction to the rate of transfer from the hydrophobic to the hydrophilic state, decreases from 1.9 days to 0.7 days. As a consequence, the mean atmospheric lifetime of $\mathrm{BC}$ decreases from 5.8 days to 5.4 days (Table 2).

[12] Midvisible aerosol optical depth (AOD), the column integrated aerosol extinction at wavelength of $550 \mathrm{~nm}$, shows a well-known pattern corresponding to the presence of anthropogenic sulfate (Figures $3 \mathrm{a}$ and $3 \mathrm{~b}$ ). Associated with the decrease of the $\mathrm{BC}$ burden, the absorption optical depth (AAOD), the column integrated aerosol extinction owing to absorption, decreases in the export region east of the Asian continent by up to 0.0004 and by about 0.0001 in the remote transport regions (Figure $3 \mathrm{c}$ ). The corresponding relative change with respect to the year 2000 case with all emissions (Figure 3d) shows that the presence of anthropogenic sulfur reduces present-day AAOD by up to $70 \%$ in the remote transport regions of the Northern Hemisphere. However, despite the fact that the $\mathrm{BC}$ burden, and therefore the mass of the dominant absorber, decreases everywhere, the AAOD shows a significant enhancement of up to 0.01 in the vicinity of the main anthropogenic source regions (Figure 3c), contributing to up to $60 \%$ of the present-day AAOD (Figure 3d). Note that the AOD and AAOD are unaffected by changes in the available diffuse radiation.

[13] The change of the black carbon column burden of the individual modes given in Figure 4 helps to unravel this complex response of the AAOD to the addition of anthropogenic sulfate. Owing to the enhanced microphysical aging, the $\mathrm{BC}$ burden in the remote transport regions decreases in all modes except the coarse mode, for which the changes (and BC burdens [cf. Stier et al., 2006a]) are generally negligible. However, over large parts of the continents, anthropogenic sulfate enhances the microphysical aging from the Aitken modes to the internally mixed 
a) Sulfate Burden
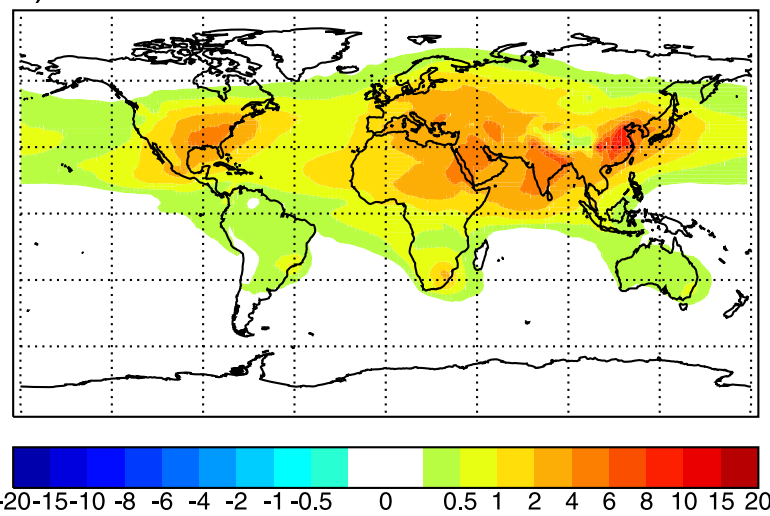

c)
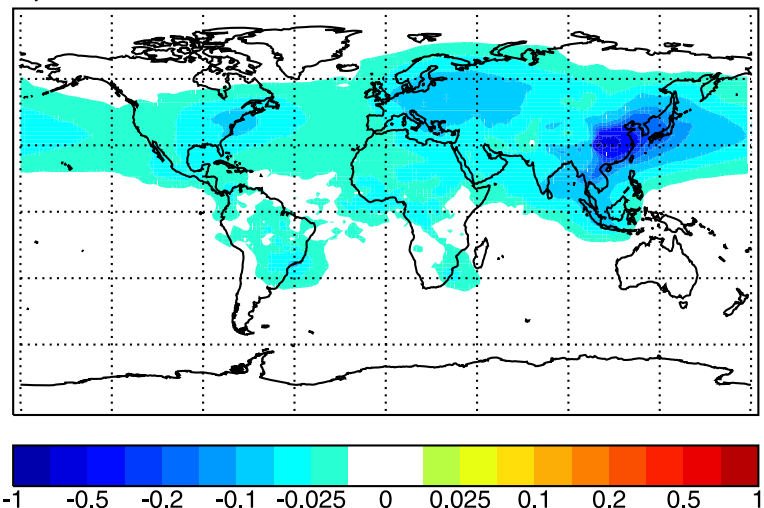

b)
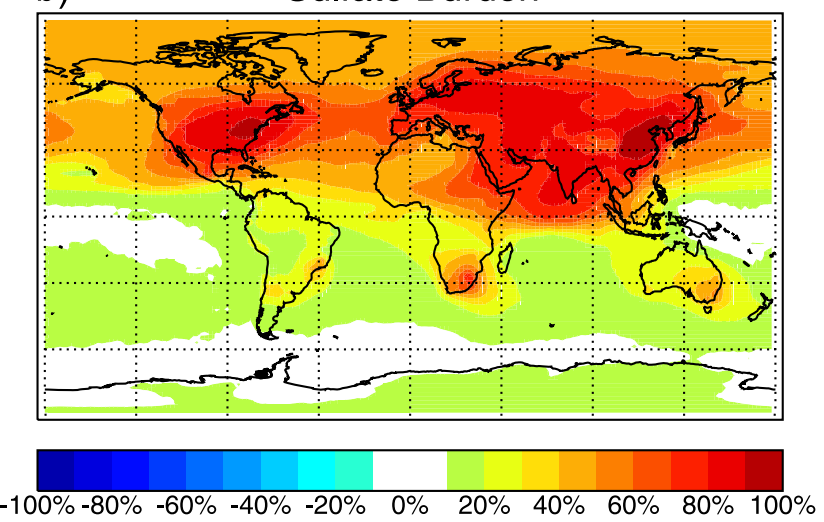

d)
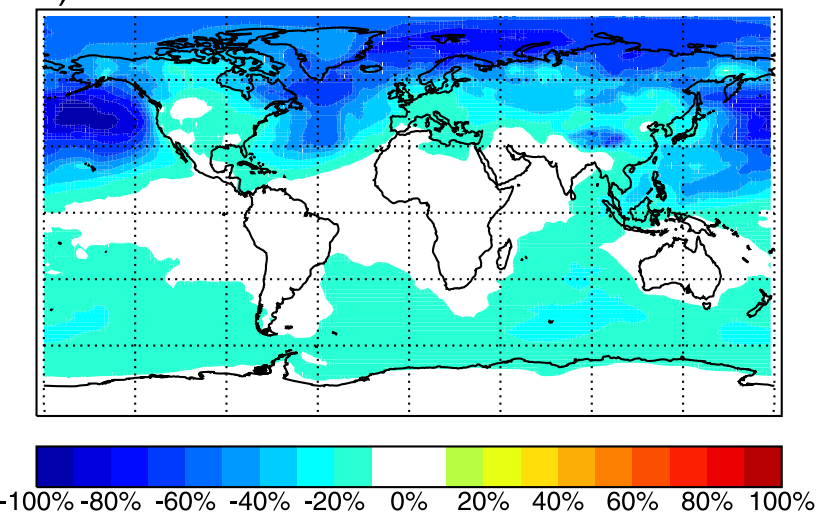

Figure 2. Annual mean aerosol column burden change of (a) sulfate $\left(\mathrm{mg}(\mathrm{S}) \mathrm{m}^{-2}\right)$, (b) sulfate (\% with respect to year 2000), (c) black carbon ( $\mathrm{mg} \mathrm{m}^{-2}$ ), and (d) black carbon (\% with respect to year 2000) due to the addition of anthropogenic sulfate; derived as difference between a year 2000 simulation with all emissions and an identical simulation without $\mathrm{SO}_{2}$ emissions from fossil fuel use, industry, and biofuels.

hydrophilic accumulation mode causing an increase of $\mathrm{BC}$ column burden in this mode (Figure 4c) despite the general decrease of the total $\mathrm{BC}$ column burden (Figure 2c). The annual mean $\mathrm{BC}$ mass fraction in this mode is generally below $10 \%$. The associated absorption enhancement outweighs the effect of the decrease of the total $\mathrm{BC}$ burden, resulting in the AAOD enhancement.

[14] These combined changes in the aerosol burden and radiative properties are reflected in the atmospheric radiative fluxes. The annual mean clear-sky top of the atmosphere radiative flux change (Figure 5a), i.e., top of the atmosphere forcing due to the addition of anthropogenic sulfur, exhibits a regional cooling of up to $-11 \mathrm{~W} \mathrm{~m}^{-2}$ and a global mean of $-0.7 \mathrm{~W} \mathrm{~m}^{-2}$. This is attributable to enhanced scattering by sulfate aerosol.

[15] The change in atmospheric absorption upon addition of anthropogenic sulfur (Figure 5b), i.e., atmospheric forcing, reflects the spatially inhomogeneous changes in the AAOD. Clear-sky atmospheric absorption is decreased by up to $1.6 \mathrm{~W} \mathrm{~m}^{-2}$ in the remote transport regions and increased by up to $7.6 \mathrm{~W} \mathrm{~m}^{-2}$ in the vicinity of the source regions. Note that the increase of absorption occurs despite a significant decrease of the $\mathrm{BC}$ burden in the same regions (Figure 2c). While this absorption enhancement associated with the increase of the AAOD could potentially be ampli- fied by an increase of diffuse and backscattered radiation owing to the increase of sulfate aerosol, this effect appears to be small as will be demonstrated in a sensitivity study in the next section. In the global annual mean, the net effect of anthropogenic $\mathrm{SO}_{2}$ on clear-sky atmospheric absorption is a decrease of $0.2 \mathrm{~W} \mathrm{~m}^{-2}$, indicating that the effect of the reduced $\mathrm{BC}$ burden resulting from a shorter lifetime overbalances the enhanced absorption due to internal mixing.

[16] The change in the surface net solar fluxes (Figure 5c), i.e., surface forcing due to the addition of anthropogenic sulfur, comprises both the dimming of the surface by enhanced backscattering of sulfate aerosol and the effects

Table 2. Annual Global Mean Black Carbon Atmospheric Lifetime $^{\mathrm{a}}$

\begin{tabular}{lccc}
\hline & Reference $^{\mathrm{b}}$ & $\begin{array}{c}\text { All Hydrophilic } \\
\text { Scavenging }\end{array}$ & $\begin{array}{c}\text { No Hydrophobic } \\
\text { Scavenging }\end{array}$ \\
\hline Year 2000 & 5.4 & 5.1 & 5.8 \\
No anthropogenic $\mathrm{SO}_{2}$ & 5.8 & 5.2 & 7.7 \\
\hline
\end{tabular}

${ }^{\mathrm{a}}$ Unit is days.

${ }^{\mathrm{b}}$ Reference scenario with reduced scavenging efficiency for the hydrophobic modes.

${ }^{c}$ Hydrophobic modes with scavenging efficiency of hydrophilic modes.

d Zero scavenging efficiency for the hydrophobic modes. 
a)
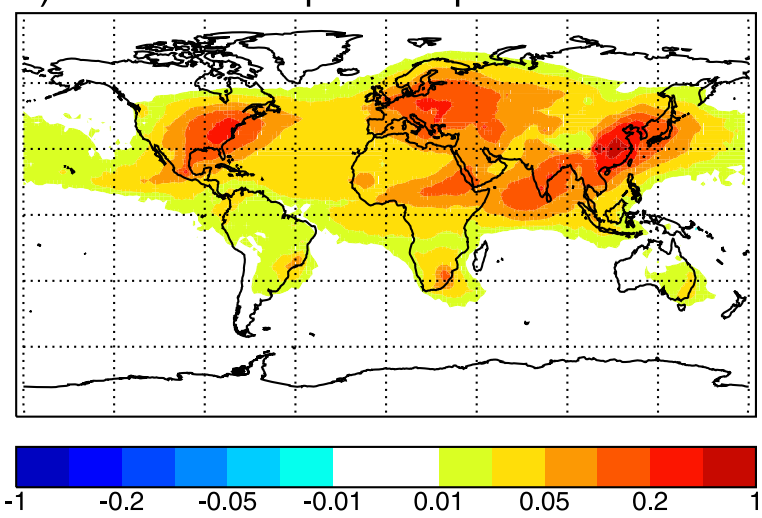

c) Absorption Optical Depth

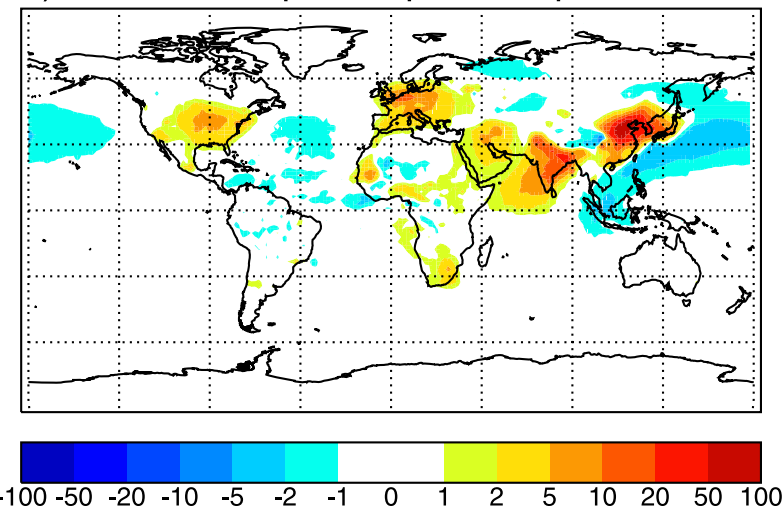

b)

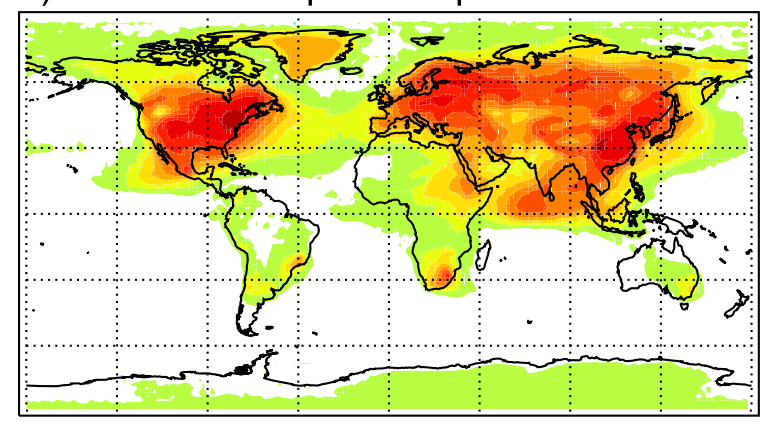

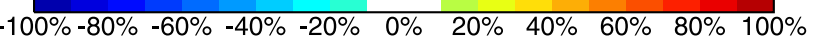

Figure 3. Annual mean change of aerosol (a) optical depth (1), (b) optical depth (\% with respect to year 2000), (c) absorption optical depth $\left(\times 10^{4}\right)$, and (d) absorption optical depth (\% with respect to year 2000$)$ at $\lambda=550 \mathrm{~nm}$ due to the addition of anthropogenic sulfate; derived as in Figure 2.

of the modulated atmospheric absorption. Therefore, in the source regions the clear-sky surface dimming due to enhanced backscattering of sulfate aerosol is further amplified by the local absorption enhancement, reaching regionally values up to $-17 \mathrm{~W} \mathrm{~m}^{-2}$. The positive surface forcing in the remote northern Pacific region, of up to $+0.5 \mathrm{~W} \mathrm{~m}^{-2}$, is the result of the decreased $\mathrm{BC}$ burden and associated absorption due to enhanced microphysical aging. The resulting global annual mean clear-sky surface forcing is $-0.5 \mathrm{~W} \mathrm{~m}^{-2}$.

\subsection{Sensitivity Studies}

[17] It has to be pointed out that the magnitude of the demonstrated effects is subject to nonnegligible uncertainties. Additional sensitivity studies are performed to investigate the importance of two key uncertainties. First, we examine the effect of replacing the calculation of the mode effective refractive indices via volume-weighted averaging of the component refractive indices by the more comprehensive Bruggeman [1935] effective medium approach (see Appendix A). Second, we analyze the sensitivity of the simulated effects to the differences in the scavenging efficiency of hydrophobic and hydrophilic modes that largely determine the effect microphysical aging exerts on the black carbon distribution. Finally, we perform additional offline radiative transfer calculations to quantify the effect of the modification of the diffuse and backscattered radiation owing to the presence of sulfate on atmospheric absorption.

[18] The main effect of replacing the calculation of the effective refractive indices from volume-weighted averaging to the Bruggeman mixing rule is to reduce the imaginary part of the effective refractive index, depending of the volume fraction of the absorber, by up to about $15 \%$ [cf. Lesins et al., 2002]. For the ECHAM5-HAM simulation of the year 2000 with all emissions, the introduction of the Bruggeman mixing rule causes a reduction in the aerosol absorption optical depth at $\lambda=550 \mathrm{~nm}$ with maxima in the anthropogenic source and biomass burning regions. However, the reduction in annual mean AAOD does not exceed $20 \%$ anywhere (not shown). Additionally, the impact of anthropogenic SU on the atmospheric absorption is calculated as difference between two simulations employing the same mixing rule so that the effect of changing the mixing rule partly cancels out. As a consequence, the overall effect of replacing the refractive index mixing rule on the impact of nonabsorbing anthropogenic aerosols on atmospheric absorption is relatively small. Thus the resulting changes in the AAOD and the atmospheric forcing are very similar to the reference scenario with volume-weighted averaging (Figure 6). The differences around the Saharan dust source region are attributable to fluctuations in the mineral dust 
a) Black Carbon - Aitken Hydrophilic
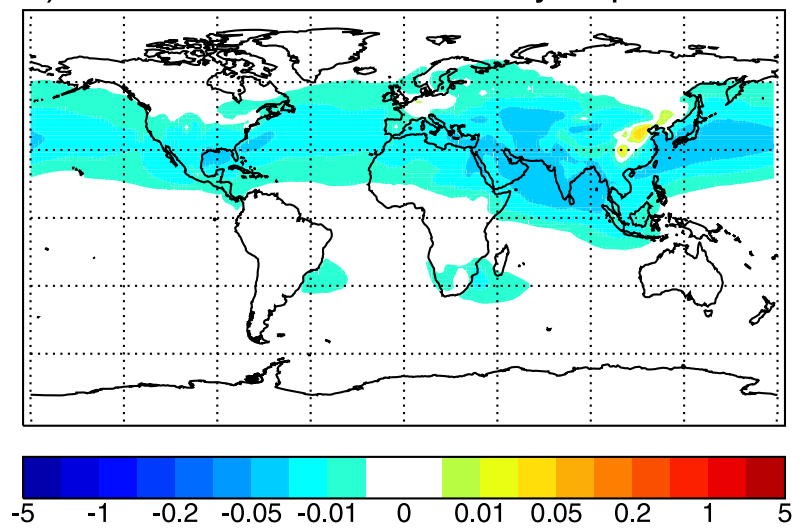

c) Black Carbon - Accumulation Hydrophilic
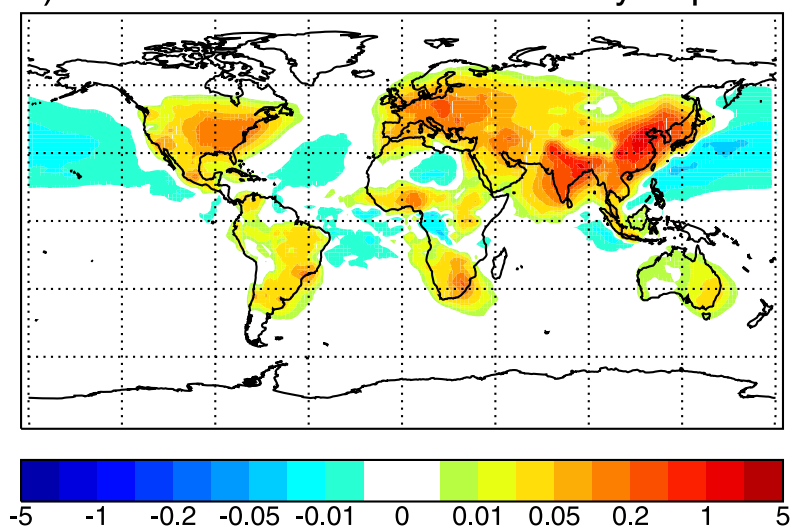

b) Black Carbon - Aitken Hydrophobic

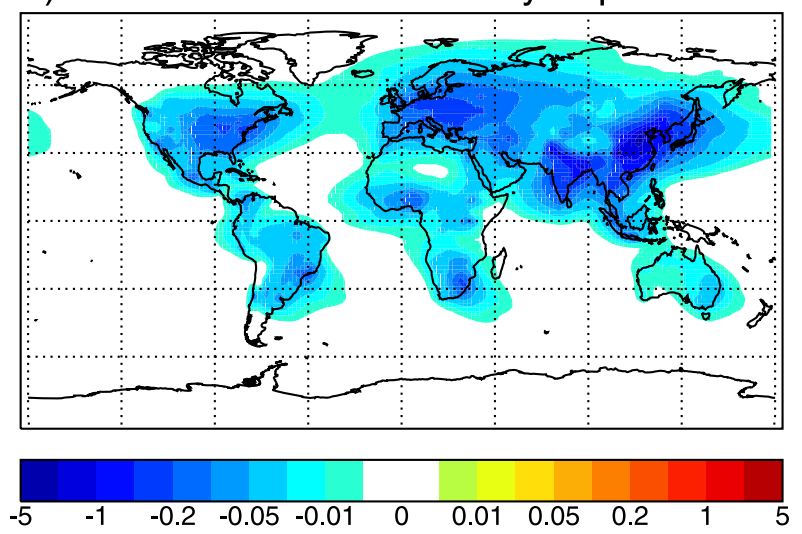

d) Black Carbon - Coarse Hydrophilic

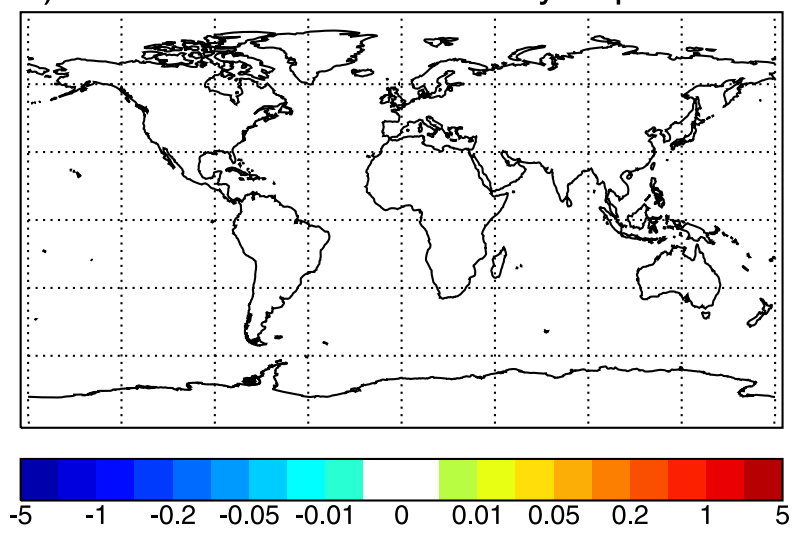

Figure 4. Annual mean black carbon column burden change of (a) hydrophilic Aitken, (b) hydrophobic Aitken, (c) hydrophilic accumulation, and (d) hydrophilic coarse mode $\left(\mathrm{mg} \mathrm{m}^{-2}\right)$ due to the addition of anthropogenic sulfate. See Table 1 for modal setup; derived as in Figure 2, so that the sum of Figures 4a$4 \mathrm{~d}$ is the total black carbon column burden change shown in Figure 2c.

burden as the nudging technique only constrains the largescale meteorology so that changes in the atmospheric absorption slightly affect the regional circulation that, in turn, alter the interactive dust emissions (not shown). The annual global mean effect of the choice of the effective medium approximation on the impact of anthropogenic SU on atmospheric absorption is negligible (Figure 6 and Table 3).

[19] Scavenging processes play a key role in the susceptibility of black carbon to aerosol aging. The (unphysical) assumption of identical scavenging efficiencies for hydrophobic and hydrophilic aerosols reduces the effect of anthropogenic SU on the BC burden as the lifetimes of the hydrophobic and hydrophilic aerosols converge. A sensitivity study ("All Hydrophilic Scavenging") is performed assuming identical scavenging efficiency for the hydrophobic modes as for the hydrophilic modes of the same size class [Stier et al., 2005], and is compared to the reference scenario in Figure 6. The effect of anthropogenic SU on the BC burden is reduced, though not completely nullified, as anthropogenic SU not only ages BC emitted into the hydrophobic Aitken mode but also grows particles from the Aitken mode size to the accumulation mode size (see Figure 4), that, accounting for nucleation scavenging, has a higher scavenging efficiency. However, the reduced effect of anthropogenic SU on the BC burden compared to the reference scenario increases the relative importance of the absorption enhancement by internal mixing. Consequently, in the global annual mean the internal mixing effect balances the effect of the reduction in the $\mathrm{BC}$ burden and renders the atmospheric forcing of anthropogenic SU negligible (Table 3).

[20] The contrary assumption of zero scavenging efficiency for hydrophobic aerosols maximizes lifetime differences between the hydrophobic and hydrophilic modes. The effect of this assumption on the impact of anthropogenic SU on atmospheric absorption is investigated in the sensitivity study ("No Hydrophobic Scavenging") applying zero scavenging efficiency for the hydrophobic modes. As evident in Figure 6, this assumption significantly enhances the effect of anthropogenic SU on the BC burden. Anthropogenic SU reduces the lifetime of $\mathrm{BC}$ from 7.7 days to 5.8 days compared to a reduction from 5.8 days to 5.4 days in the reference scenario (Table 2). As a consequence, the absorption reduction associated with the $\mathrm{BC}$ burden reduction is significantly stronger than in the reference scenario. Thus the absorption enhancement owing to internal mixing only in the main source regions outweighs the effect of the burden reduction, indicated by small areas of positive AAOD and atmospheric forcing. This has a distinct effect 

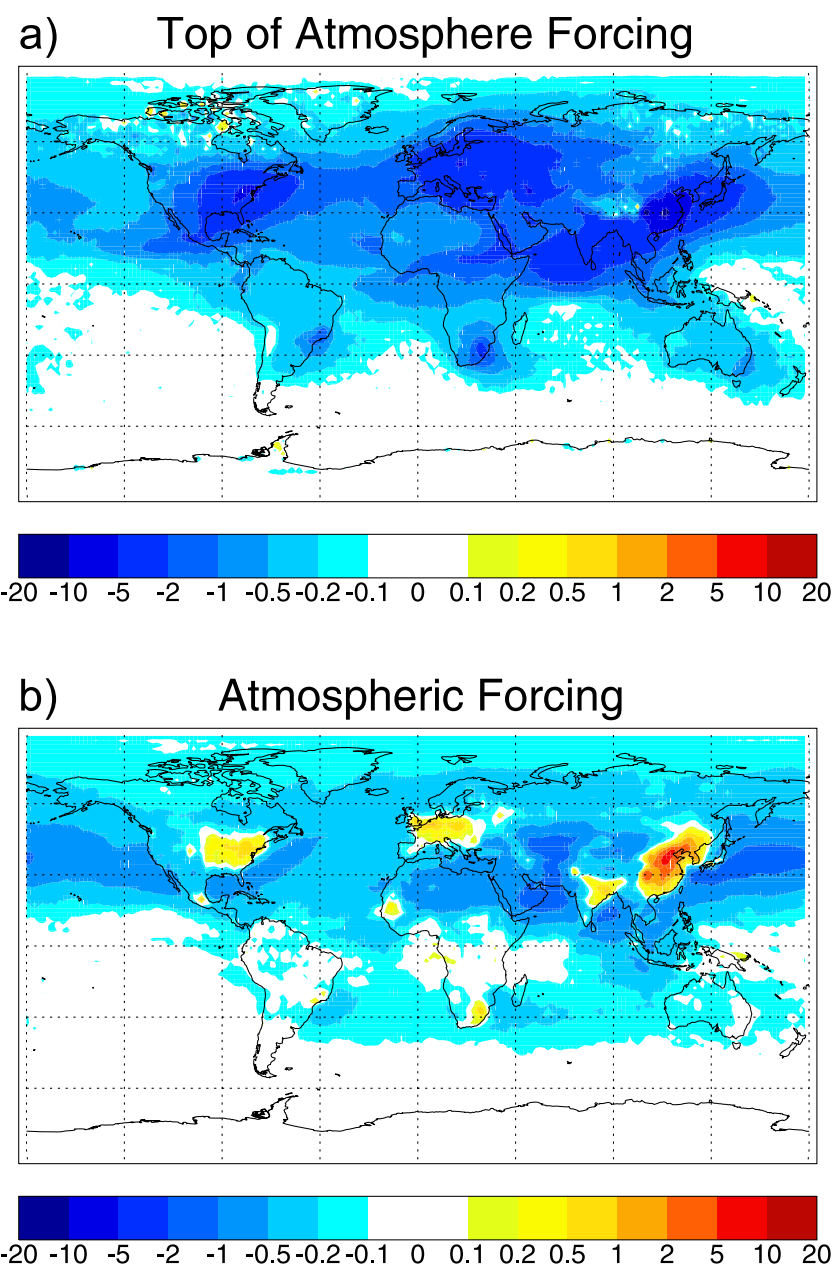

c)
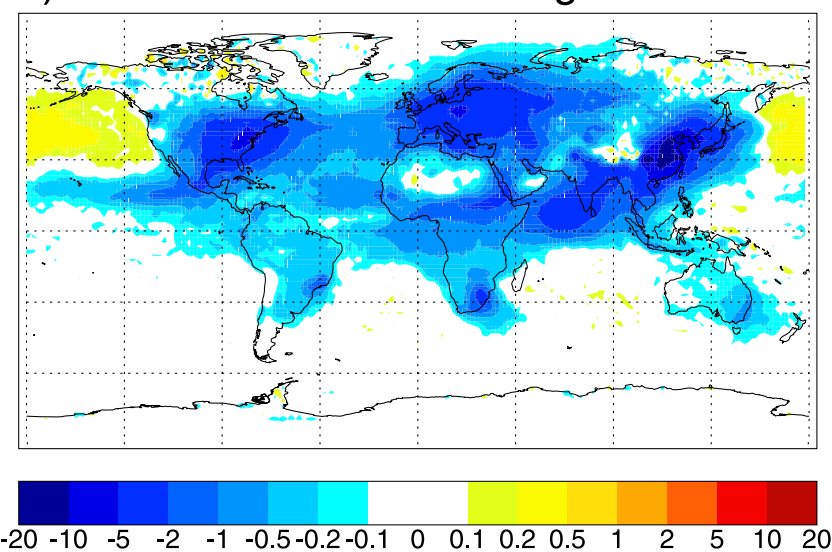

Figure 5. Annual mean clear-sky shortwave (a) top of the atmosphere forcing, (b) atmospheric forcing, and (c) surface forcing $\left(\mathrm{W} \mathrm{m} \mathrm{m}^{-2}\right)$ due to the addition of anthropogenic sulfate; derived as in Figure 2 but for the shortwave top of the atmosphere radiative flux, atmospheric absorption, and surface radiative flux, respectively.

on the surface forcing induced by anthropogenic sulfate. While the surface forcing, as in the reference scenario, remains negative in the main anthropogenic source and export regions, it is positive for the remote transport regions so that its global annual mean is reduced to $-0.17 \mathrm{~W} \mathrm{~m}^{-2}$, compared to $-0.52 \mathrm{~W} \mathrm{~m}^{-2}$ in the reference scenario (Table 3). This strong effect of reducing the scavenging efficiency of the hydrophobic modes to zero can be explained largely by the fact that, besides microphysical aging, scavenging constitutes the dominant sink process of the hydrophobic Aitken mode, as dry deposition and sedimentation are inefficient for this size range.

[21] Additional offline radiative transfer calculations are performed to quantify the effect of an increase in the diffuse and backscattered radiation owing to the presence of sulfate on atmospheric absorption. The calculations are performed with two one-dimensional radiative transfer models, employing the higher accuracy adding-doubling method and the two-stream approximation also used in the radiation module of ECHAM5-HAM. The lowest $2 \mathrm{~km}$ of a clear-sky atmospheric column at $45^{\circ} \mathrm{N}$ in the summer season are initialized with lognormal aerosol populations of $\mathrm{SU}$ and $\mathrm{BC}$ with a SU/BC mass ratio of 10 and number median radii of $0.08 \mu \mathrm{m}$ and $0.22 \mu \mathrm{m}$ and standard deviations of 1.7 and 1.3 , respectively. The BC aerosol population has an AOD at $550 \mathrm{~nm}$ of 0.035 and the atmospheric absorption without the influence of SU, calculated with the adding-doubling method, is $14.5 \mathrm{~W} \mathrm{~m}^{-2}$. Adding SU as externally mixed aerosol population to increase the total AOD to 0.15 enhances the atmospheric absorption by $0.3 \mathrm{~W} \mathrm{~m}^{-2}$. In contrast, adding the same SU aerosol population and assuming a complete internal mixture of BC into the sulfate particles increases the total AOD to 0.13 and enhances the atmospheric absorption by $7.2 \mathrm{~W} \mathrm{~m}^{-2}$, employing the volume weighted mixing rule. For the two-stream approximation, the absorption enhancement by the introduction of $\mathrm{SU}$ as external mixture is below the accuracy of the approach. A comparably small absorption enhancement by the presence of externally mixed sulfate of less than $10 \%$ has been found by N. Bellouin (personal communication, 2006) in a similar simulation set up employing the Streamer radiative transfer model [Key and Schweiger, 1998]. Thus the effect of sulfate on the atmospheric absorption via the modification of the radiative fluxes by scattering appears to be a second-order effect compared to the effect of internal mixing on the radiative properties.

\section{Conclusions}

[22] Absorption of solar radiation by aerosols, in particular by black carbon, is well established as of importance for regional and global climate. Previous studies of the effect of nonabsorbing aerosols, primarily of sulfate, on atmospheric absorption have focused on either of two phenomena: (1) Sulfate microphysically ages initially hydrophobic black carbon to a hydrophilic state and therefore reduces its lifetime and abundance, decreasing atmospheric absorption, and (2) internal mixing of nonabsorbing aerosols, such as sulfate, with black carbon increases the absorption efficiency of black carbon and therefore increases atmospheric absorption. Additionally, the presence of scattering aerosols increases the amount of diffuse solar radiation available for absorption. In this work, we have analyzed the effects of anthropogenic sulfate on atmospheric absorption with a comprehensive aerosol-climate model with prognostic treatment of the aerosol composition, 

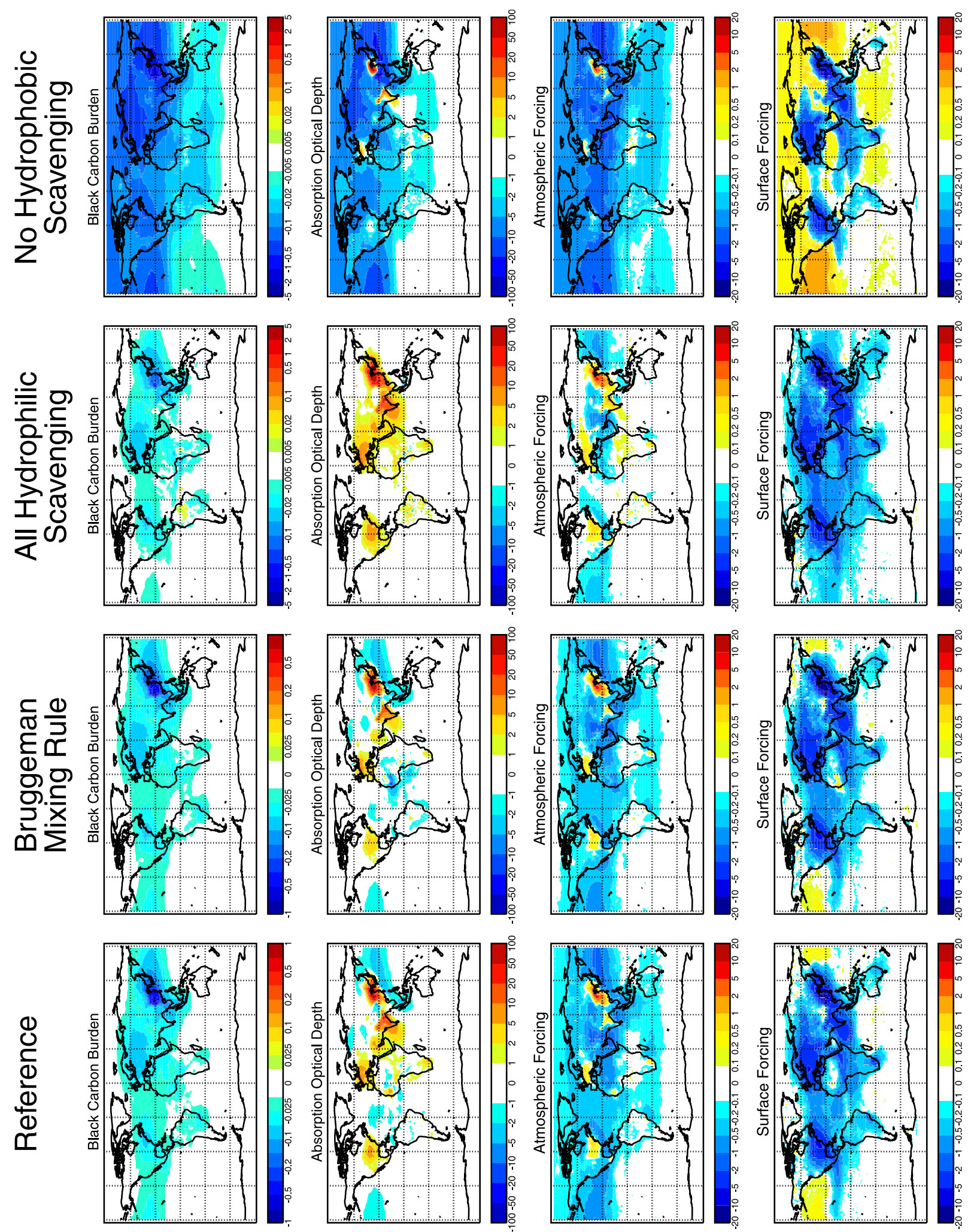
Table 3. Annual Global Mean Clear-Sky Shortwave Forcings Due to the Addition of Anthropogenic Sulfate ${ }^{\mathrm{a}}$

\begin{tabular}{lccc}
\hline & Reference $^{\mathrm{b}}$ & Bruggeman Mixing Rule $^{\mathrm{c}}$ & All Hydrophilic Scavenging $^{\mathrm{d}}$ \\
\hline Top of the atmosphere forcing & -0.72 & -0.70 & -0.68 \\
Atmospheric forcing & -0.20 & -0.18 & -0.02 \\
Surface forcing & -0.52 & -0.52 & -0.66 \\
\hline${ }^{\mathrm{a}}$ Unit is W mophobic Scavenging \\
${ }^{-2}$.
\end{tabular}

size distribution, and mixing state, allowing the concurrent investigation of these mechanisms. Our findings demonstrate their significance on the global scale, with a regional character depending on the distance from the aerosol sources.

[23] The effects of anthropogenic sulfate are isolated as the difference between a year 2000 simulation with the complete year 2000 emissions and an identical simulation without $\mathrm{SO}_{2}$ emissions from fossil fuel use, industry, and biofuels. In the reference scenario the mode effective refractive indices are calculated on the basis of volume averaging of the component refractive indices and residual scavenging efficiencies for the hydrophobic modes are assumed, accounting for unresolvable subgrid-scale aging and the neglect of chemical aging processes.

[24] The introduction of anthropogenic sulfate enhances the microphysical aging and, owing to increased wet deposition, reduces the atmospheric burden of black carbon. Consequently, the clear-sky atmospheric absorption in the remote transport regions is decreased by up to $1.6 \mathrm{~W} \mathrm{~m}^{-2}$, regionally allowing more radiation to reach the surface, constituting a positive surface forcing. However, internal mixing of black carbon with anthropogenic sulfate and the associated enhancement of the absorption efficiency increase clear-sky atmospheric absorption in the source regions by up to $7.6 \mathrm{~W} \mathrm{~m}^{-2}$, despite a concurrent decrease of the black carbon burden. A comparable enhancement is also simulated employing a more elaborate approach for the calculation of the effective mode refractive indices, the Bruggeman [1935] mixing rule.

[25] In the global annual mean, the net effect of anthropogenic sulfate on atmospheric absorption by black carbon is a decrease of $0.2 \mathrm{~W} \mathrm{~m}^{-2}$, indicating that, globally, the effect of the reduced black carbon burden overbalances the absorption enhancement due to internal mixing. However, anthropogenic sulfate introduces significant regional perturbations and gradients of absorption, with potential effects on regional climate.

[26] While the general impact of anthropogenic sulfate on atmospheric absorption, the enhancement close to the source regions and the reduction in the remote transport regions, appears to be a robust feature, the magnitude of the demonstrated effects and the regional distribution of the transition between the two regimes are sensitive to the scavenging efficiency difference between the hydrophobic and hydrophilic aerosol modes. In a sensitivity study with the (unphysical) assumption of identical scavenging efficiencies for the hydrophobic and hydrophilic modes of the same size class, the effect of anthropogenic sulfate on the black carbon burden is significantly reduced. Therefore the associated absorption reduction becomes in the global annual mean balanced by the absorption enhancement by internal mixing. Contrary, for a sensitivity study maximizing the susceptibility of the black carbon burden to anthropogenic sulfate by assuming zero scavenging efficiency for the hydrophobic modes, the black carbon burden and absorption reductions are significantly enhanced relative to the reference scenario so that the effect on the atmospheric absorption is intensified to $-0.6 \mathrm{~W} \mathrm{~m}^{-2}$, compared to $-0.2 \mathrm{~W} \mathrm{~m}^{-2}$ in the reference scenario. Consequently, the absorption reduction in the remote transport regions outweighs the dimming of solar radiation by anthropogenic sulfate resulting in large areas of positive surface forcing so that the global annual mean surface forcing by anthropogenic sulfate is reduced to $-0.2 \mathrm{~W} \mathrm{~m}^{-2}$, compared to $-0.5 \mathrm{~W} \mathrm{~m}^{-2}$ in the reference scenario. While the neglect of subgrid-scale and chemical aging processes let the assumption of zero scavenging efficiencies for the hydrophobic modes seem a strong assumption, it is in line with previous modeling studies [e.g., Cooke et al., 1999].

[27] Advanced parameterizations, applicable at the scales of global modeling, as well as more detailed information about the near-source aerosol mixing state are required to improve the quantitative estimate of the impact of anthropogenic sulfate on atmospheric absorption. Additional uncertainties include the fact that in this study secondary aerosols other than sulfate are represented by a primary equivalent (organic matter) or neglected (nitrate), leading to an underestimate of the amount of condensable vapors of both natural and anthropogenic origin. The relevance of the enhancement of black carbon absorption by the internal mixture with secondary organic aerosol (SOA) has been demonstrated in a laboratory study by Schnaiter et al. [2005]. The microphysical aging of atmospheric aerosols

Figure 6. Annual mean change of black carbon column burden $\left(\mathrm{mg} \mathrm{m}^{-2}\right)$, absorption optical depth $\left(\times 10^{4}\right)$ at $\lambda=550 \mathrm{~nm}$, clear-sky shortwave atmospheric forcing $\left(\mathrm{W} \mathrm{m}^{-2}\right)$, and clear-sky shortwave surface forcing ( $\left.\mathrm{W} \mathrm{m}^{-2}\right)$ due to the addition of anthropogenic sulfate. Reference, reference scenario with volume-weighted averaging of refractive indices and reduced scavenging efficiency for the hydrophobic modes; Bruggeman Mixing Rule, calculation of mode effective refractive indices based on the Bruggeman mixing rule; All Hydrophilic Scavenging, hydrophobic modes with scavenging efficiency of hydrophilic modes; No Hydrophobic Scavenging, zero scavenging efficiency for the hydrophobic modes. 
by SOA has been investigated in a measurement study by Maria et al. [2004]. Their derived microphysical aging timescale owing to SOA of about 4-8 days is significantly slower than model derived estimates of the microphysical aging timescale owing to sulfate, that range in order of $0.3-$ 1.7 days [Wilson et al., 2001; Riemer et al., 2004; Stier et al., 2005]. While these results indicate a prominent role of sulfate for microphysical aging, differences in the methodologies and definitions as well as the fact that the Maria et al. [2004] estimate is based on a very limited sample size need to emphasized. Additional direct measurements of the evolution of the atmospheric black carbon mixing state are essential to constrain and improve model estimates of aerosol absorption. Finally, observations show that black carbon particles have complex nonspherical morphologies upon emission, depending on the source type and combustion temperature [Mikhailov et al., 2006; Andreae and Gelencsér, 2006], affecting microphysical aging [van Poppel et al., 2005] and radiative properties [Martins et al., 1998a]. Microphysical aging processes and subsequent internal mixing transform these structures to more spherical morphologies, supporting the spherical approximation and application of Mie theory in large-scale modeling [Martins et al., 1998b; Mikhailov et al., 2006]. Additional observational efforts are required to determine the atmospheric evolution of the black carbon morphology and to quantify the errors associated with the currently applied spherical approximation in global forcing estimates. However, while these uncertainties demand for continuous research, they do not alter the qualitative understanding of the mechanisms nor their large-scale regional patterns identified in this study.

[28] Our findings have important implications for future emission scenarios and mitigation strategies. Variations in sulfur emissions affect atmospheric solar absorption despite the fact that sulfate is virtually nonabsorbing. The effects are spatially highly inhomogeneous; thereby the sulfur to black carbon emission ratio plays an important role. The processes involved are complex so that comprehensive global microphysical aerosol models are required to constrain the regional and global implications of future emission changes.

\section{Appendix A: Effective Medium Approaches}

[29] In the standard setup of HAM [Stier et al., 2005] the refractive indices for internally mixed modes are calculated from volume-weighted averaging of the refractive indices of the aerosol components. However, as discussed by Chýlek et al. [1995] and Lesins et al. [2002] the volume-weighted mixing of the refractive indices for strongly absorbing inclusions, such as BC, in a less absorbing medium, such as $\mathrm{SU}$, results in an overestimation of the effective imaginary part of the refractive index compared to an average of explicit calculations over all possible positions of the inclusion in the host medium. Improved approximations of the mode effective refractive indices can be obtained from mixing rules derived from effective medium approaches, quantifying the macroscopic radiative properties as average over the microscopic arrangement of the components.
[30] A general formulation for mixing rules of the dielectric constants (square of the refractive indices) of $n$ components can be expressed as [Aspnes, 1982]:

$$
\frac{\varepsilon_{\text {eff }}-\varepsilon_{0}}{\varepsilon_{\text {eff }}+2 \varepsilon_{0}}=\sum_{k=1}^{n} f_{k} \frac{\varepsilon_{k}-\varepsilon_{0}}{\varepsilon_{k}+2 \varepsilon_{0}}
$$

where $\varepsilon_{\text {eff }}$ is the effective dielectric constant of the mixture, $\varepsilon_{0}$ is the dielectric constant of the host medium, and $\varepsilon_{k}$ and $f_{k}$ are dielectric constant and volume fraction of the component $k$, respectively.

[31] While the Maxwell-Garnet mixing rule [Garnett, $1904,1906]$ is more accurate for the representation of an insoluble core in a soluble host medium [Lesins et al., 2002], it requires the explicit choice of a host and inclusion medium and is not valid for cores with large volume fractions. Thus the implementation for aerosol modules with complex internally mixed modes of varying volume fractions, such as HAM, is not straightforward.

[32] The Bruggeman [1935] mixing rule requires no explicit choice of the core and host media as it is assumed that the components are embedded in a host medium of the effective dielectric constant, i.e., $\varepsilon_{0}=\varepsilon_{\text {eff, }}$, so that the left hand side in equation (A1) vanishes, reducing it to an implicit equation for $\varepsilon_{\text {eff: }}$ :

$$
\sum_{k=1}^{n} f_{k} \frac{\varepsilon_{k}-\varepsilon_{\text {eff }}}{\varepsilon_{k}+2 \varepsilon_{\text {eff }}}=0
$$

The resulting real and imaginary parts of the refractive indices lie generally in between the results of the two possible choices of host and inclusion media of the Maxwell-Garnet mixing rule [Chýlek et al., 2000] and both approaches, as well as assumption of a concentric core shell structure, agree well with results from more explicit calculations [Chýlek et al., 1995]. For the application in the model, the complex refractive indices of the components [Stier et al., 2005] are converted to the corresponding complex dielectric constants for which equation (A2) is solved iteratively employing the Newton-Raphson method. From a one year test simulation it was established that the method converges for every grid point and time step after a maximum of 6 iterations, so that a fixed number of 7 iterations is applied globally.

[33] Acknowledgments. This research was supported by the NASA Earth Observing System Interdisciplinary Science Program (NASA EOSIDS) and by the German Ministry for Education and Research (BMBF) under the DEKLIM Project. Olivier Boucher was supported by the Climate Prediction Programme of the UK Department for Environment, Food, and Rural Affairs. The simulations were performed at the German High Performance Computing Centre for Climate- and Earth System Research. We would also like to thank Nicolas Bellouin (Met Office, Exeter, UK) for supporting radiative transfer calculations and Michael Schulz (LSCE, Gifsur-Yvette, France) for helpful discussions.

\section{References}

Ackerman, T. P., and O. B. Toon (1981), Absorption of visible radiation in atmosphere containing mixtures of absorbing and non-absorbing particles, Appl. Opt., 20, 3661-3668.

Andreae, M. O., and A. Gelencsér (2006), Black carbon or brown carbon? The nature of light-absorbing carbonaceous aerosols, Atmos. Chem. Phys. Disc., 6, 3419-3463.

Aspnes, D. E. (1982), Local-field effects and effective-medium theory-A microscopic perspective, Am. J. Phys., 50, 704-709.

Bruggeman, D. (1935), Calculation of various physics constants in heterogeneous substances, i dielectric constants and conductivity of mixed bodies from isotropic substances, Ann. Phys., 24, 636-664. 
Charlson, R. J., and M. J. Pilat (1969), Climate: The influence of aerosols, J. Appl. Meteorol., 8, 1001-1002.

Chung, S. H., and J. H. Seinfeld (2002), Global distribution and climate forcing of carbonaceous aerosols, J. Geophys. Res., 107(D19), 4407, doi:10.1029/2001JD001397.

Chung, S. H., and J. H. Seinfeld (2005), Climate response of direct radiative forcing of anthropogenic black carbon, J. Geophys. Res., 110, D11102, doi:10.1029/2004JD005441.

Chýlek, P., G. Videen, D. Ngo, R. G. Pinnick, and J. D. Klett (1995), Effect of black carbon on the optical properties and climate forcing of sulfate aerosols, J. Geophys. Res., 100, 16,325-16,332.

Chýlek, P., G. Videen, W. D. J. Geldart, J. S. Dobbie, and H. C. W. Tso (2000), Effective medium approximations for heterogeneous particles, in Light Scattering by Nonspherical Particles: Theory, Measurements, and Applications, edited by M. I. Mishchenko et al., pp. 273-308, Elsevier, New York.

Cooke, W. F., C. Liousse, H. Cachier, and J. Feichter (1999), Construction of a $1^{\circ} \times 1^{\circ}$ fossil fuel emission data set for carbonaceous aerosol and implementation and radiative impact in the ECHAM4 model, J. Geophys. Res., 104, 22,137-22,162.

Croft, B., U. Lohmann, and K. von Salzen (2005), Black carbon aging in the Canadian Centre for Climate Modelling and Analysis atmospheric general circulation model, Atmos. Chem. Phys., 5, 1931-1949.

Dentener, F., et al. (2006), Emissions of primary aerosol and precursor gases for the years 2000 and 1750: Prescribed data-sets for AeroCom, Atmos. Chem. Phys. Disc., 6, 2703-2763.

Feichter, J., E. Kjellstrom, H. Rodhe, F. Dentener, J. Lelieveld, and G. J. Roelofs (1996), Simulation of the tropospheric sulfur cycle in a global climate model, Atmos. Environ., 30, 1693-1707.

Fouquart, Y., and B. Bonnel (1980), Computations of solar heating of the Earth's atmosphere: A new parameterization, Beitr. Phys. Atmos., 53 , $35-62$.

Garnett, J. C. M. (1904), Colours in metal glasses and in metallic films, Philos. Trans. R. Soc. London, 203, 385-420.

Garnett, J. C. M. (1906), Colours in metal glasses, in metallic films, and in metallic solutions-II, Philos. Trans. R. Soc. London, 205, 237-288.

Hess, M., P. Koepke, and I. Schult (1998), Optical properties of aerosols and clouds: The software package OPAC, Bull. Am. Meteorol. Soc., 79, $831-844$

Jacobson, M. Z. (2000), A physically-based treatment of elemental carbon optics: Implications for global direct forcing of aerosols, Geophys. Res. Lett., 27, 217-220.

Jacobson, M. Z. (2001), Strong radiative heating due to the mixing state of black carbon in atmospheric aerosols, Nature, 409, 695-697.

Jacobson, M. Z., and J. H. Seinfeld (2004), Evolution of nanoparticle size and mixing state near the point of emission, Atmos. Environ., 38, 18391850 .

Key, J. R., and A. J. Schweiger (1998), Tools for atmospheric radiative transfer: Streamer and fluxnet, Comput. Geosci., 24, 443-451.

Kiehl, J. T., and B. P. Briegleb (1993), The relative roles of sulfate aerosols and greenhouse gases in climate forcing, Science, 260, 311-314.

Koch, D. (2001), Transport and direct radiative forcing of carbonaceous and sulfate aerosols in the GISS GCM, J. Geophys. Res., 106, 20,31120,332 .

Langner, J., and H. Rodhe (1991), A global 3-dimensional model of the tropospheric sulfur cycle, J. Atmos. Chem., 13, 225-263.

Lesins, G., P. Chylek, and U. Lohmann (2002), A study of internal and external mixing scenarios and its effect on aerosol optical properties and direct radiative forcing, J. Geophys. Res., 107(D10), 4094, doi:10.1029/ 2001JD000973

Lin, S. J., and R. B. Rood (1996), Multidimensional flux-form semiLagrangian transport schemes, Mon. Weather Rev., 124, 2046-2070.

Lohmann, U., and E. Roeckner (1996), Design and performance of a new cloud microphysics scheme developed for the ECHAM general circulation model, Clim. Dyn., 12, 557-572.

Maria, S. F., L. M. Russell, M. K. Gilles, and S. C. B. Myneni (2004), Organic aerosol growth mechanisms and their climate-forcing implications, Science, 306, 1921-1924.

Martins, J. V., P. Artaxo, C. Liousse, J. S. Reid, P. V. Hobbs, and Y. J. Kaufman (1998a), Effects of black carbon content, particle size, and mixing on light absorption by aerosols from biomass burning in Brazil, J. Geophys. Res., 103, 32,041-32,050.

Martins, J. V., P. V. Hobbs, R. E. Weiss, and P. Artaxo (1998b), Sphericity and morphology of smoke particles from biomass burning in Brazil, J. Geophys. Res., 103, 32,051-32,057.

Menon, S., J. Hansen, L. Nazarenko, and Y. F. Luo (2002), Climate effects of black carbon aerosols in China and India, Science, 297, $2250-2253$

Mikhailov, E. F., S. S. Vlasenko, I. A. Podgorny, V. Ramanathan, and C. E. Corrigan (2006), Optical properties of soot-water drop agglomerates: An experimental study, J. Geophys. Res., 111, D07209, doi:10.1029/ 2005JD006389.

Mlawer, E. J., S. J. Taubman, P. D. Brown, M. J. Iacono, and S. A. Clough (1997), Radiative transfer for inhomogeneous atmospheres: RRTM, a validated correlated-k model for the longwave, J. Geophys. Res., 102, $16,663-16,682$.

Morcrette, J.-J., S. A. Clough, E. J. Mlawer, and M. J. Iacono (1998) Impact of a validated radiative transfer scheme, RRTM, on the ECMWF model climate and 10-day forecasts, Tech. Memo. 252, 48 pp., Eur. Cent. for Med.-Range Weather Forecasts, Reading, U. K. (Available at http:// www.ecmwf.int)

Myhre, G., F. Stordal, T. F. Berglen, J. K. Sundet, and I. S. A. Isaksen (2004), Uncertainties in the radiative forcing due to sulfate aerosols, J. Atmos. Sci., 61, 485-498.

Nakicenovic, N. (2000), Greenhouse gas emissions scenarios, Technol. Forecast. Soc. Change, 65, 149-166.

Nordeng, T. E. (1994), Extended versions of the convective parameterization scheme at ECMWF and their impact on the mean and transient activity of the model in the tropics, Tech. Memo. 206, 42 pp., Eur. Cent for Med.-Range Weather Forecasts, Reading, U. K. (Available at http:// www.ecmwf.int)

Penner, J. E., et al. (2001), Aerosols, their direct and indirect effects, in Climate Change 2001: The Scientific Basis, edited by J. T. Houghton et al., pp. 289-348, Cambridge Univ. Press, New York.

Riemer, N., H. Vogel, and B. Vogel (2004), Soot aging time scales in polluted regions during day and night, Atmos. Chem. Phys., 4, $1885-$ 1893

Roeckner, E., et al. (2003), The atmospheric general circulation model ECHAM5. Part I: Model description, Rep. 349, Max Planck Inst. for Meteorol., Hamburg, Germany. (Available at http://www.mpimet.mpg.de) Roeckner, E., P. Stier, J. Feichter, S. Kloster, M. Esch, and I. Fischer-Bruns (2006), Impact of carbonaceous aerosol forcing on regional climate change, Clim. Dyn., doi:10.1007/s00382-006-0147-3, in press.

Schnaiter, M., C. Linke, O. Mohler, K. H. Naumann, H. Saathoff, R. Wagner, U. Schurath, and B. Wehner (2005), Absorption amplification of black carbon internally mixed with secondary organic aerosol, J. Geophys. Res., 110, D19204, doi:10.1029/2005JD006046.

Simmons, A. J., and J. K. Gibson (2000), The ERA-40 project plan, ERA40 Proj. Rep. Ser. 1, 62 pp., Eur. Cent. for Med.-Range Weather Forecasts, Reading, U. K. (Available at http://www.ecmwf.int/)

Stier, P. (2005), Towards the assessment of the aerosol radiative effectsA global modeling approach, Rep. Earth Syst. Sci., 9, 111 pp., MaxPlanck-Inst. for Meteorol., Hamburg, Germany. (Available at http:/ www.mpimet.mpg.de/)

Stier, P., et al. (2005), The aerosol-climate model ECHAM5-HAM, Atmos. Chem. Phys., 5, 1125-1156.

Stier, P., J. Feichter, E. Roeckner, S. Kloster, and M. Esch (2006a), The evolution of the global aerosol system in a transient climate simulation from 1860 to 2100, Atmos. Chem. Phys., 6, 3059-3076.

Stier, P., J. Feichter, S. Kloster, E. Vignati, and J. Wilson (2006b), Emission-induced nonlinearities in the global aerosol system: Results from the ECHAM5-HAM aerosol-climate model, J. Clim., 19(16), 3845-3862.

Tiedtke, M. (1989), A comprehensive mass flux scheme for cumulus parameterization in large-scale models, Mon. Weather Rev., 117, 1779-1800.

Tompkins, A. M. (2002), A prognostic parameterization for the subgridscale variability of water vapor and clouds in large-scale models and its use to diagnose cloud cover, J. Atmos. Sci., 59, 1917-1942.

van Poppel, L. H., H. Friedrich, J. Spinsby, S. H. Chung, J. H. Seinfeld, and P. R. Buseck (2005), Electron tomography of nanoparticle clusters: Implications for atmospheric lifetimes and radiative forcing of soot, Geophys. Res. Lett., 32, L24811, doi:10.1029/2005GL024461.

Vignati, E., J. Wilson, and P. Stier (2004), M7: An efficient size-resolved aerosol microphysics module for large-scale aerosol transport models, J. Geophys. Res., 109, D22202, doi:10.1029/2003JD004485.

Weingartner, E., H. Burtscher, and U. Baltensperger (1997), Hygroscopic properties of carbon and diesel soot particles, Atmos. Environ., 31, $2311-$ 2327

Wilson, J., C. Cuvelier, and F. Raes (2001), A modeling study of global mixed aerosol fields, J. Geophys. Res., 106, 34,081-34,108.

Zuberi, B., K. S. Johnson, G. K. Aleks, L. T. Molina, and A. Laskin (2005), Hydrophilic properties of aged soot, Geophys. Res. Lett., 32, L01807, doi:10.1029/2004GL021496.

O. Boucher, Hadley Centre, Met Office, Exeter EX1 3PB, UK

J. Feichter and S. Kinne, Atmosphere in the Earth System, Max Planck Institute for Meteorology, D-20146 Hamburg, Germany.

J. H. Seinfeld and P. Stier, Department of Environmental Science and Engineering, California Institute of Technology, Pasadena, CA 91125 , USA. (philip.stier@caltech.edu) 